\title{
Modelación de un ensayo de corte directo en arena mediante el método de elementos discretos tridimensional
}

\author{
Modelling of a direct shear test in sand using the 3D Discrete Element Method
}

\section{Antonio Salazar, Esteban Sáez y Gislaine Pardo}

Departamento de Ingeniería Estructural y Geotécnica, Pontificia Universidad Católica de Chile, Vicuña Mackenna 4860, Macul, Santiago,Chile, afsalaza@uc.cl, esaez@ing.puc.cl,gspardo@uc.cl

En el presente trabajo se presenta un ensayo de corte directo sobre una arena gruesa, modelado usando el Método de Elementos Discretos tridimensionales. Esta metodología representa el suelo como un conjunto de partículas, reproduciendo su respuesta macroscópica mediante las interacciones micro-mecánicas entre partículas. El modelo DEM consistió en aproximadamente 70000 partículas perfectamente esféricas. El tamaño de las partículas es consistente con la granulometría real de la arena ensayada. Se exploraron varias metodologías con el fin de reproducir un índice de vacios inicial similar al de laboratorio. Los resultados numéricos del modelo fueron comparados con los resultados de ensayos experimentales de una arena gruesa proveniente de etapas previas de este estudio. El objetivo fue el de reproducir precisamente la respuesta macroscópica obtenida en el ensayo de laboratorio con el modelo de elementos discretos, y estudiar el efecto de los micro-parámetros en la relación tensióndeformación, así como en la dilatación del material. Se estudió además la migración de partículas fuera del plano de corte, así como los aspectos micromecánicos ligados a la formación de la banda de corte característica de este tipo de ensayos. Los resultados muestran que, al incluir un modelo de resistencia a la rotación para simular la forma de las partículas, la respuesta en tensión puede ser reproducida adecuadamente con este método, pero la dilatancia característica de una arena densa es muy dificil de replicar.

Palabras clave: método elementos discretos tridimensionales, granulometría real, modelo de resistencia a la rotación
In the present article, a direct shear test was modelled using the $3 D$ Discrete Element Method. This approach describes the soil as an assembly of particles, reproducing the macroscopic soil behaviour from micro-mechanics interactions between individual particles. About 70000 particles were used in a $3 D$ model, developed to reproduce a direct shear test. The numerical model results will be compared with experimental tests of a coarse sand from previous stages of this research. The real material was modeled as perfect individual spheres, with sizes consistent with the real grain size distribution and with a rolling friction approach to include the sand's grain shape. Several methodologies were followed in order to reproduce an initial void ratio similar to those obtained experimentally. The goal is to accurately reproduce the macro-scale response of the laboratory tests with the DEM model, and to study the effect of the micro-mechanical parameters in the stress path and the material deformation. Two specimens with different initial void ratio were modelled to study the effect of the micro-mechanical parameters and the initial fabric compactness on the model results. Afterwards, a model calibrated against experimental data is presented with a brief analysis of the particle migration and the contact orientation. The results show the development of a clear shear zone in the middle portion of the specimens, with an evident out of plane migration of particles. It is shown that, including a rolling resistant model, the stress path can be appropriately reproduced, but the dilatancy characteristic of a dense sand was very difficult to replicate.

Keywords: 3D Discrete Element Method DEM, real grain size distribution, rolling resistant model

\section{Introducción}

En este trabajo se desarrolló un modelo del ensayo de corte directo utilizando el Método de los Elementos Discretos
DEM en su versión tridimensional, de forma de investigar la habilidad de esta técnica para reproducir la respuesta de una arena limpia. El ensayo consiste en una pequeña caja de 
dos hemisferios, al inferior se le impone un desplazamiento controlado mientras que el superior permanece estático, registrándose la fuerza de reacción. Sobre la parte superior se le aplica una fuerza de confinamiento vertical y su desplazamiento es monitoreado.

Se decidió implementar la versión tridimensional del método DEM puesto que la versión plana no predice adecuadamente el ángulo de fricción máximo y residual bajo condiciones de corte (Fleischamann et al., 2013), debido a la migración fuera del plano de las partículas entre otros efectos. Para poder considerar la granulometría real del material en el modelo, los granos de arena se simularon como esferas perfectas con un modelo de fricción a la rotación para representar el efecto de la forma (Wensrich y Katterfeld, 2012).

El efecto que los parámetros micro-mecánicos tienen sobre el comportamiento al corte del material, se estudiaron a través de un análisis de sensibilidad. Además, dos probetas fueron modeladas con diferente índice de vacíos inicial, para de esta forma poder también analizar el efecto de la compacidad inicial sobre la respuesta. Finalmente, se realizó una nueva probeta con parámetros calibrados para reproducir la respuesta experimental real de la arena (Pardo, 2013; Pardo y Sáez, 2014). También se presenta la red de fuerzas y se ilustra la migración de partículas que tiene lugar fuera del plano de aplicación del corte.

\section{Desarrollo del modelo de Elementos Dis- cretos}

\section{Descripción de la arena gruesa ensayada}

Las propiedades mecánicas más importantes de la arena ensayada se muestran en la Tabla 1, como se puede observar se trata de una arena con un poco de cohesión. Las propiedades de resistencia al corte fueron obtenidas de ensayos de corte directo a tres diferentes confinamientos: 40, 80 y $160 \mathrm{kPa}$. La información de los transductores de fuerza y desplazamiento obtenida de estos ensayos de corte, se utilizaron para comparar los resultados de los modelos numéricos.

La Figura 1a muestra los bordes aparentes de diferentes granos de la arena ensayada, obtenidos a partir de fotografías tomadas con un microscopio y utilizando para su procesamiento el paquete Balu para Matlab (Mery, 2011). Bajo un análisis visual, las partículas tienen una esfericidad cercana a los 0.8 y una redondez de 0.5 aproximadamente, lo cual corresponde a una arena de partículas de forma subredondeada. Según el análisis granulométrico de la Figura $1 \mathrm{~b}$, la arena tiene menos de $1 \%$ de material fino, quedando retenida prácticamente toda su masa en cuatro tamices y siendo el № 16 el más grande (partículas entre 2.16 y 1.18 $\mathrm{mm}$ de diámetro).

Tabla 1: Propiedades de la arena real

\begin{tabular}{|l|c|}
\hline Propiedad & Valor \\
\hline Densidad $\rho, \mathrm{kg} / \mathrm{m}^{3}$ & 1578 \\
\hline Índice de vacíos $e_{0}$ & 0.679 \\
\hline Porosidad $n_{0}$ & 0.405 \\
\hline Ángulo de fricción $\phi^{\prime},{ }^{\circ}$ & 35 \\
\hline Cohesión $c^{\prime}, \mathrm{kPa}$ & 8 \\
\hline
\end{tabular}

a)

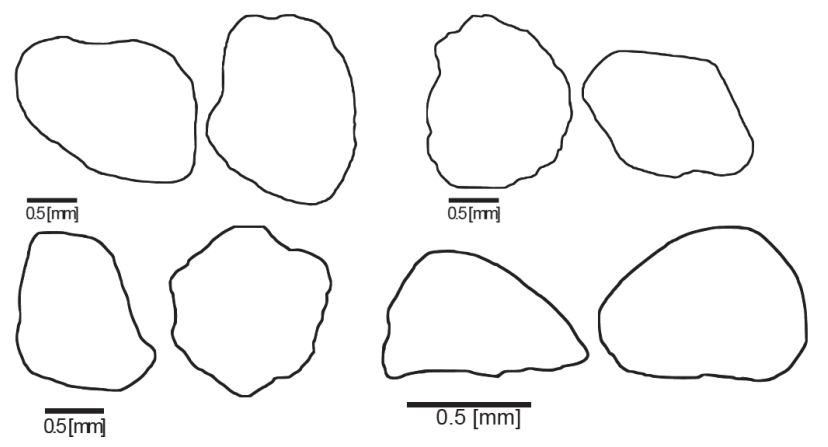

b)

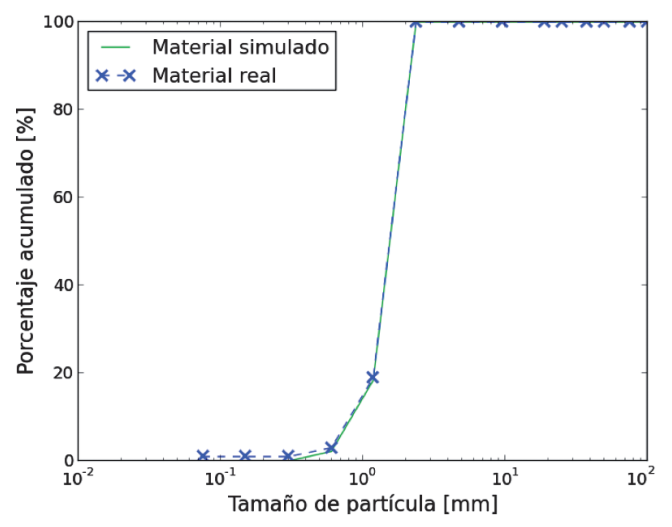

Figura 1: a) Bordes de los granos de arena y b) distribución granulométrica real y simulada

\section{Breve descripción del Método de Elementos Discretos}

La principal ventaja de este método, es que simula el material como un conjunto de partículas individuales que interactúan entre sí, lo que permite capturar el movimiento relativo y rotaciones de todos los granos que componen el medio, sin la necesidad de un modelo constitutivo sofisticado. Debido a la independencia de los grados de libertad se pueden modelar grandes deformaciones, sin 
mayor dificultad. Para aplicaciones con medios granulares, el equilibrio dinámico queda determinado por la segunda ley de Newton en (1) y en (2), donde $M_{i}$ e $I_{i}$ son la masa y la inercia de la $i$-ésima partícula, $F^{i j}$ y $M^{i j}$ son la fuerza de contacto inducida por la partícula $j$ sobre la partícula $i$, y el momento que induce esta fuerza. $F_{e}$ es la fuerza producto de cualquier campo externo (sólo la gravedad es considerada en este trabajo) y $M_{e}$ el momento respectivo debido a la fuerza $F_{e}$.

$$
\begin{aligned}
& M_{i} \ddot{x}_{i}=\sum_{j} F^{i j}+F_{e} \\
& I_{i} \ddot{\theta}_{i}=\sum_{j} M^{i j}+M_{e}
\end{aligned}
$$

El tiempo es dividido en pequeños intervalos, donde los desplazamientos y rotaciones son calculados a través de la doble integración de (1) y (2). Para evitar inestabilidad del sistema y asegurar la convergencia del modelo, se debe adoptar un paso de tiempo pequeño. En este estudio, el paso de tiempo máximo se considera como el mínimo entre los criterios de Hertz y de Rayleigh (Li et al., 2005). El modelo fue implementado utilizando el programa LIGGGHTS (Kloss et al., 2012), el cual es un código de Elementos Discretos especialmente adaptado para resolver problemas con materiales granulares e implementado para trabajar en paralelo. Mayor información se puede encontrar en el sitio web de los desarrolladores.

\section{Modelación de los contactos intergranulares}

El modelo más usado para simular la fuerza de repulsión cuando dos partículas se encuentran, y el adoptado en esta investigación, es el modelo basado en la teoría de contacto de Hertz (1882), expresión (3). Esta teoría utiliza las propiedades elásticas del material (módulo de Young equivalente $\bar{E}$ ) y el área de contacto para calcular la fuerza normal. Debido a que el modelo considera el área de contacto, la relación es no lineal con la interpenetración $\delta_{n}$ y con el radio equivalente $\bar{R}$.

$$
\begin{aligned}
& F_{n}=\left(\frac{4}{3} \bar{E} \sqrt{\bar{R}}\right) \delta_{n}^{1.5} \\
& \frac{1}{\bar{E}}=\frac{\left(1-v_{i}^{2}\right)}{E_{i}}+\frac{\left(1-v_{j}^{2}\right)}{E_{j}} \\
& \frac{1}{\bar{R}}=\frac{1}{R_{i}}+\frac{1}{R_{j}}
\end{aligned}
$$

La magnitud de la fuerza tangencial está determinada por la teoría de Mindlin (1949) y limitada por el criterio de fricción de Coulomb, según (6). De esta forma, si la fuerza tangencial supera el criterio determinado por la fuerza normal $F_{n}$ y el coeficiente de fricción $\mu$, las partículas deslizan relativamente entre sí.

$F_{\max }^{t} \leq\left|\mu F_{n}\right|$

Como los granos se modelaron como esferas perfectas, adicionalmente se aplicó el modelo de fricción a la rotación de Ai et al. (2011) para considerar el efecto de la forma de los granos. Este modelo adiciona un momento al equilibrio dinámico de cada partícula que ayuda a estabilizar la rotación. El modelo se controla a través de dos parámetros: el coeficiente de amortiguamiento viscoso a la rotación $\eta \mathrm{y}$ el coeficiente de fricción a la rotación $\mu_{r}$. La razón para utilizar este tipo de modelos recae en que la orientación de la fuerza de contacto no siempre pasa por el centroide de las partículas (lo que se asume implícitamente cuando se utilizan esferas perfectas), como se ilustra en la Figura 2.

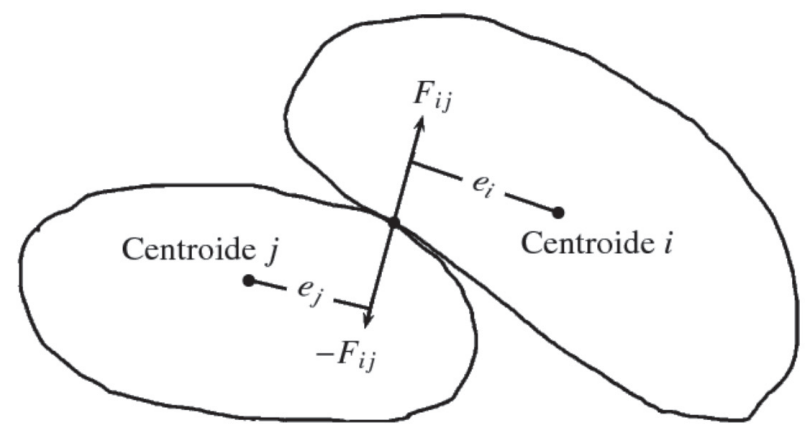

Figura 2: Contacto entre granos reales

\section{Distribución del tamaño de las partículas simuladas}

El tamaño de las partículas simuladas es completamente acorde con la granulometría real, como se puede ver en la Figura $1 b$. En esta figura, también se puede observar que el porcentaje más fino (inferior al 1\%) no fue modelado, puesto que considerar estas partículas incrementa considerablemente el costo computacional, sin tener una mayor influencia en la respuesta macroscópica del modelo. El mismo porcentaje de masa de arena retenida en cada uno de los tamices ASTM fue simulado con partículas 
cuyo diámetro fue distribuido linealmente entre la abertura del tamiz donde quedaron retenidas y la abertura del tamiz superior, como se pude observar en la Figura 3a. El 1\% más fino que no fue modelado fue distribuido en los otros tamices, y es por esto que en la Figura $3 \mathrm{~b}$ el porcentaje simulado acumulado en cada tamiz es ligeramente superior al porcentaje real.

(a)

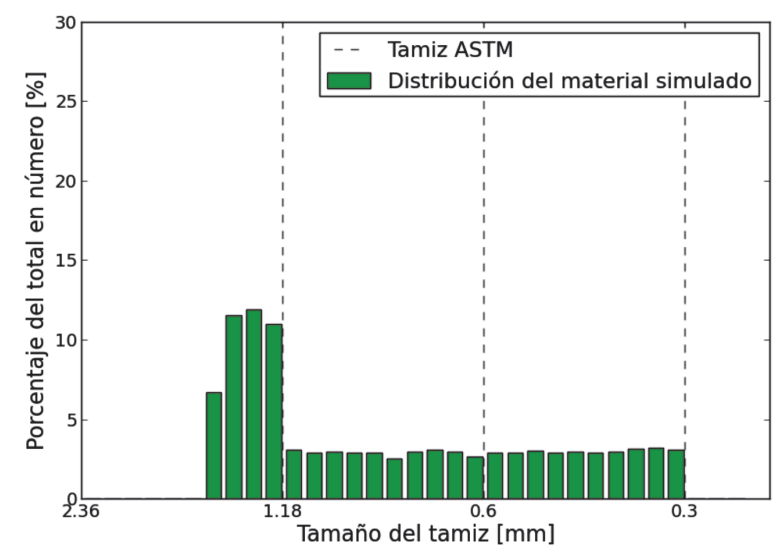

(b)

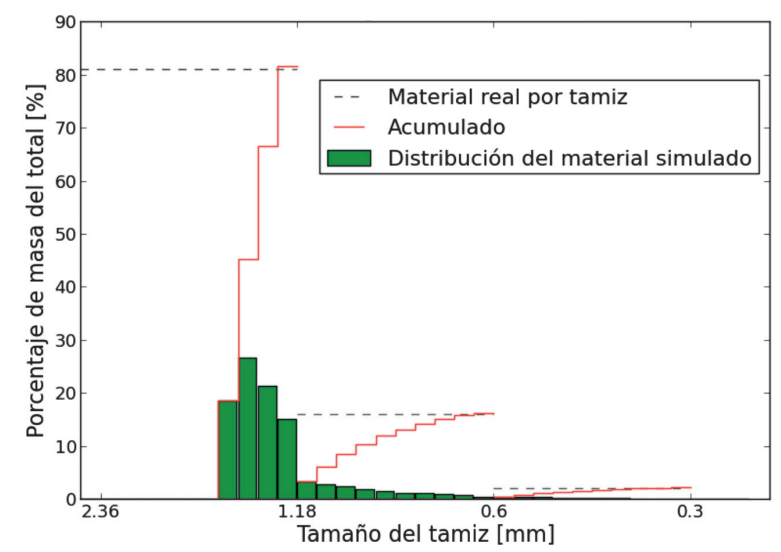

Figura 3: a) Histograma del número de partículas simuladas y b) histograma de partículas por masa

\section{Condiciones de borde y generación del conjunto de partículas}

Todas las paredes reales del ensayo, excepto la superior, fueron modeladas como bordes rígidos. Este tipo de condición de borde no tiene inercia y su movimiento es controlado a través de la imposición de la velocidad. Estos bordes rígidos repelen las partículas usando también la teoría de Hertz según (3). El borde superior fue simulado como un borde servo-controlado, el cual actúa igual que un borde rígido, pero la diferencia es que su velocidad es manejada automáticamente a través de un algoritmo para mantener una presión objetivo constante sobre las partículas.

Cada una de las probetas simuladas se compone por 70000 partículas aproximadamente, las cuales se generan aleatoriamente en el espacio en tres capas de $0.8 \mathrm{~cm}$ cada una. Una vez que las partículas de la capa son creadas, se dejan caer libremente producto de la gravedad hasta que se alcanza el equilibrio. Este proceso es repetido 3 veces para llenar la caja de corte hasta una altura de 2.4 $\mathrm{cm}$. Para finalizar con el proceso de asentamiento se activa el borde servo-controlado y se confinan las partículas. Finalmente, se impone una velocidad a todos los bordes de la mitad inferior y se detienen cuando se ha alcanzado una deformación de $8 \mathrm{~mm}$. El modelo desarrollado descrito anteriormente se puede observar en la Figura 4.

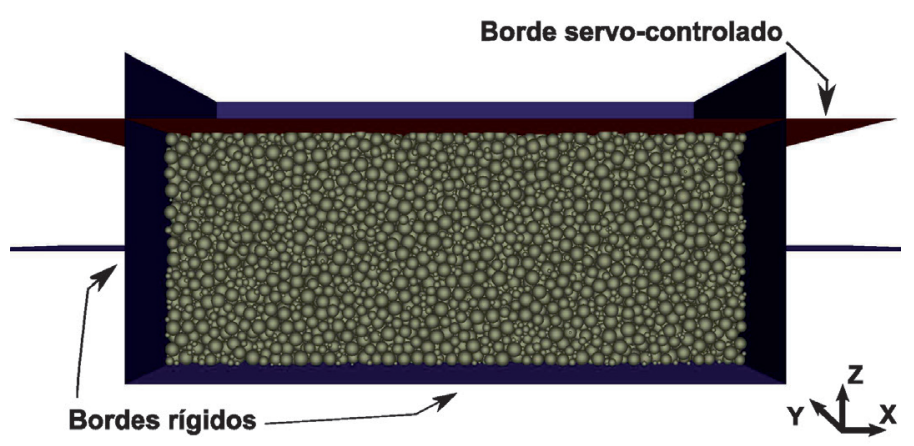

Figura 4: Modelo DEM implementado

Para controlar el índice de vacíos inicial $e_{0}$, y así obtener probetas densas o sueltas, diferentes coeficiente de fricción inicial $\mu_{i}$ fueron utilizados. Entre más bajo el valor de $\mu_{i}$ utilizado, mayor es la densidad de la probeta. Antes de comenzar el proceso de corte, se modifican los valores de $\mu, \mu_{r}$ y $\eta$ para simular el comportamiento de la arena. No se utiliza el modelo friccional rotacional durante el proceso de fabricación de la probeta.

\section{Micro-parámetros en la respuesta macros- cópica}

Dos probetas diferentes (A y B) fueron modeladas para investigar los efectos de los parámetros micro-mecánicos en la respuesta macroscópica, cuyas propiedades se indican en la Tabla 2. La diferencia entre estas dos probetas es que se utilizaron diferentes coeficientes de fricción inicial $\mu_{i}$, consecuentemente se obtienen diferentes índices de vacíos iniciales $e_{0 \mathrm{i}}$. El peso específico de la fase sólida $\gamma_{\mathrm{s}}$ fue 
obtenido a partir de pruebas de laboratorio, mientras que los módulos de Young $E$ y coeficiente de Poisson $v$ fueron elegidos de tal forma de tener una rigidez de contacto realista, según (3).

Tabla 2: Propiedades de las probetas simuladas

\begin{tabular}{|l|c|c|c|}
\hline Propiedad & Probeta A & Probeta B & Probeta C \\
\hline$\gamma_{\mathrm{s}, \mathrm{kg} / \mathrm{m}^{3}}$ & 2650 & 2650 & 2650 \\
\hline$E, \mathrm{~kg} / \mathrm{m}^{2}$ & $5 \cdot 10^{8}$ & $5 \cdot 10^{8}$ & $5 \cdot 10^{9}$ \\
\hline$\nu$ & 0.256 & 0.256 & 0.256 \\
\hline$\mu_{i}$ & 0 & 0.3 & 0.3 \\
\hline$\eta_{i}$ & 0 & 0 & 0 \\
\hline$\mu_{r i}$ & 0 & 0 & 0 \\
\hline$\mu$ & $0.3-0.6$ & $0.3-0.6$ & 0.3 \\
\hline$\eta$ & $0.3-0.6$ & $0.3-0.6$ & 0.1 \\
\hline$\mu_{r}$ & $0.3-0.6$ & $0.3-0.6$ & 0.4 \\
\hline$e_{0 \mathrm{i}}$ obtenido & 0.489 & 0.594 & 0.643 \\
\hline
\end{tabular}

En la Figura 5 se muestra la respuesta del material para diferentes combinaciones del coeficiente de fricción $\mu$, el coeficiente de amortiguamiento viscoso a la rotación $\eta \mathrm{y}$ el coeficiente de fricción a la rotación $\mu_{r}$, durante la fase de corte. Se puede observar en la comparación de tensión de la Figura 5 a que el coeficiente de fricción $\mu$ tiene influencia tan sólo a pequeñas deformaciones, tendiendo a incrementar sólo el valor máximo de tensión. Para deformaciones mayores a $4 \mathrm{~mm}$ (gran deformación), todas las curvas tienen aproximadamente el mismo valor, lo que indica que tan sólo con el uso del coeficiente de fricción de Coulomb no se puede controlar la respuesta a grandes deformaciones. Con respecto a la deformación volumétrica, el incremento del valor de este parámetro aumenta la dilatación de la muestra sin cambiar considerablemente la forma de la curva, obteniéndose una configuración más suelta conforme crece el valor de este parámetro (Figura 5).

Los resultados del análisis efectuado para el coeficiente de amortiguamiento viscoso a la rotación $\eta$ se muestran en la Figura 5b. El efecto de este parámetro en la respuesta no es muy perceptible, solamente se puede notar un leve incremento de la tensión máxima y del volumen a gran deformación. El efecto más importante del coeficiente de fricción a la rotación $\mu_{r}$ (Figura $5 \mathrm{c}$ ), es que aumenta la tensión residual. También aumenta el valor máximo de tensión al inicio de la etapa de corte, pero de menor manera que $\mu$. Al igual que los otros dos parámetros, se puede observar que $\mu_{r}$ incrementa la deformación volumétrica del material. Como este parámetro simula la forma del grano, un mayor valor de este parámetro representa un grano más anguloso, por lo que se simula una mayor trabazón entre partículas lo que se manifiesta como una tensión máxima algo mayor y como un aumento de la deformación volumétrica. En términos generales, la variación volumétrica del material a lo largo del proceso de corte fue muy difícil de replicar.

En las tres comparaciones se aprecia que la probeta A tiene una mayor tensión a baja deformación, efecto típico de una arena relativamente densa, mientras que la respuesta tensional de la probeta B se asemeja bastante a la de una arena relativamente suelta. La probeta A tiene una pendiente inicial mayor que la probeta $\mathrm{B}$ y tiene un mayor desplazamiento vertical de la tapa superior que la probeta más suelta. Estos dos efectos también se comprueban experimentalmente debido a la mayor densidad de contactos, lo cual aumenta la rigidez.

\section{Comparación del modelo numérico calibra- do con resultados de laboratorio}

Con la información recopilada en el análisis de sensibilidad, se construyó una tercera probeta con parámetros calibrados, los cuales se muestran en la Tabla 2, junto con el índice de vacíos obtenido. Esta probeta se implementó con una mayor rigidez de contacto, para así tener una mayor pendiente inicial, lo que a su vez causa un mayor índice de vacíos debido a que las interpenetraciones son menores. E1 índice de vacíos de la probeta calibrada difiere levemente del valor real, puesto que la forma no esférica de los granos reales tiende a reducir el índice de vacíos. Para un confinamiento de $160 \mathrm{kPa}$, el modelo puede reproducir fielmente la curva tensión-deformación durante todo el proceso de corte (Figura 6). Para los confinamientos de 80 y $40 \mathrm{kPa}$, el modelo puede reproducir la tensión residual, pero no el máximo inicial, esto se puede deber a que en el laboratorio se lograron diferentes índices de vacíos iniciales. La dilatancia del material aumenta con la disminución del confinamiento, lo que también sucede en el laboratorio. En todos los confinamientos la curva de desplazamiento se parece cualitativamente a los datos de 
(a)
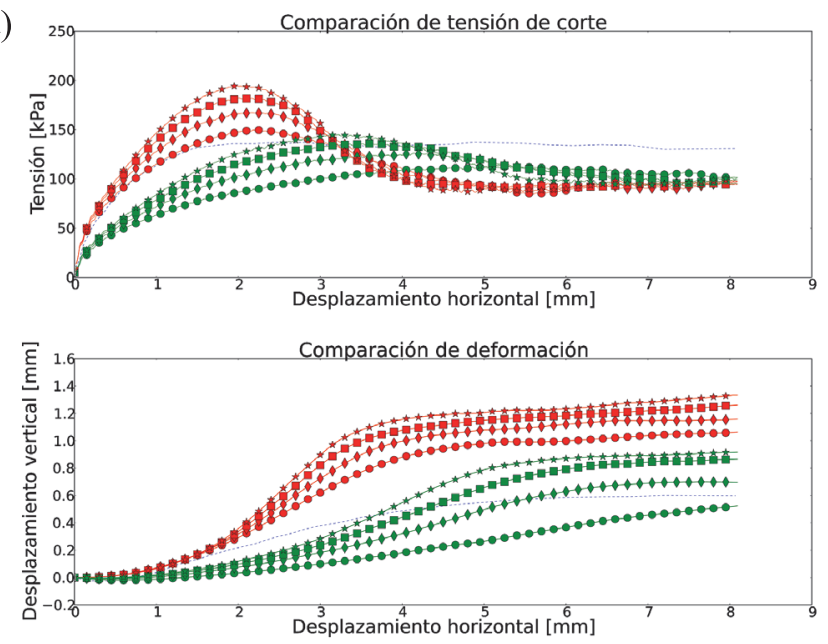

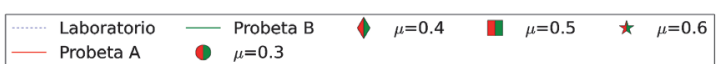

(b)
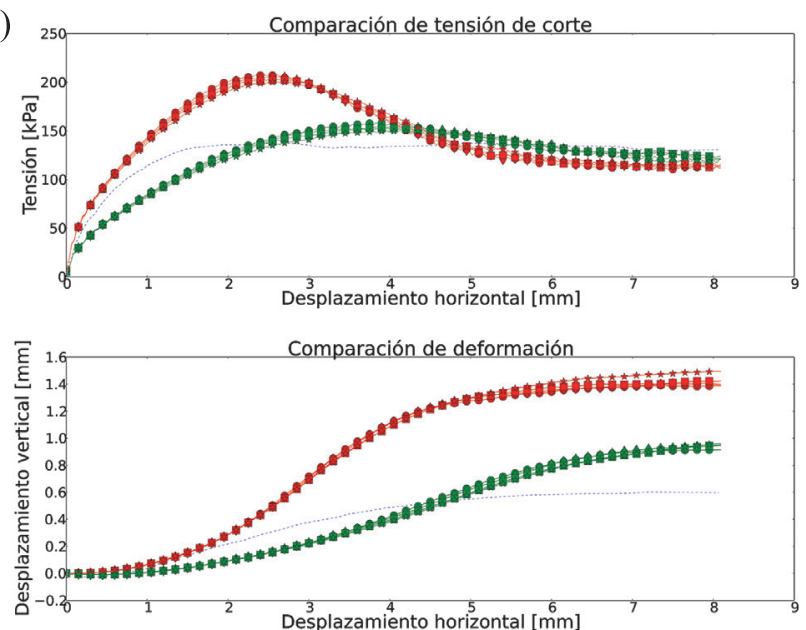

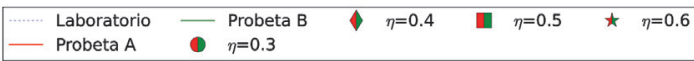

(c)
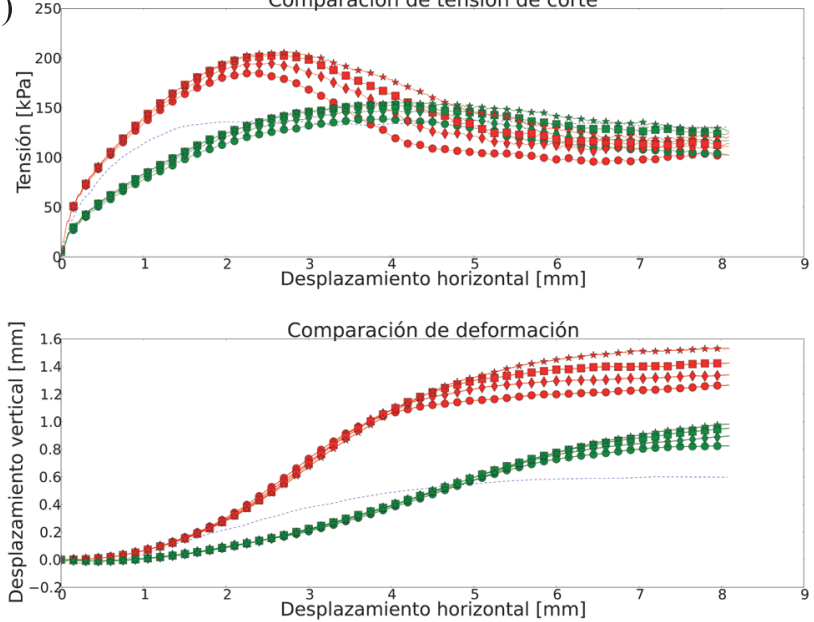

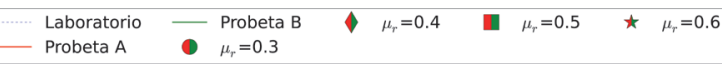

Figura 5: Análisis de sensibilidad de los parámetros de DEM, influencia de a) $\mu\left(\mu_{\mathrm{r}}=0.2 ; \eta=0.2\right)$, b) $\eta\left(\mu=0.4 ; \mu_{\mathrm{r}}=0.5\right)$ y c $)$ $\mu_{\mathrm{r}}(\mu=0.4 ; \eta=0.5)$ (a)
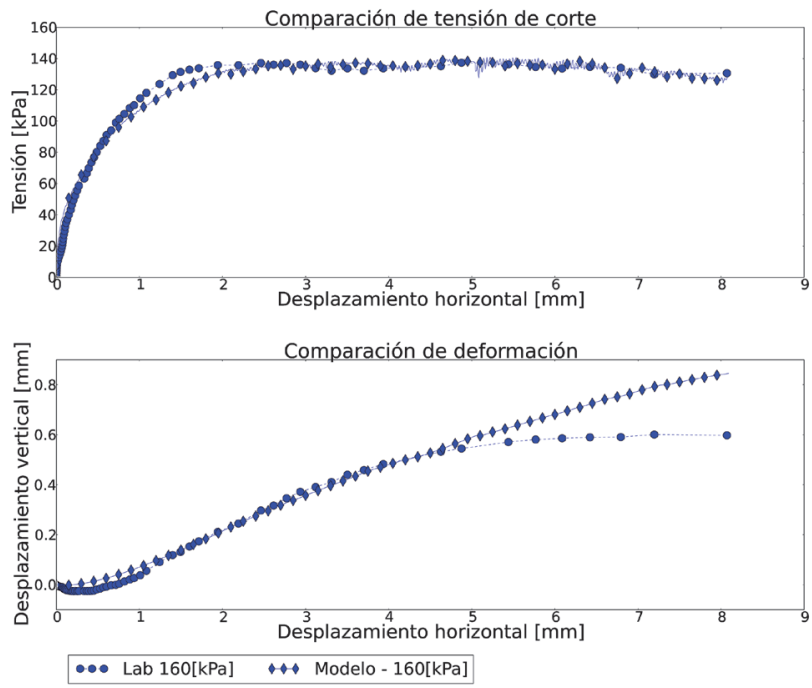

(b)
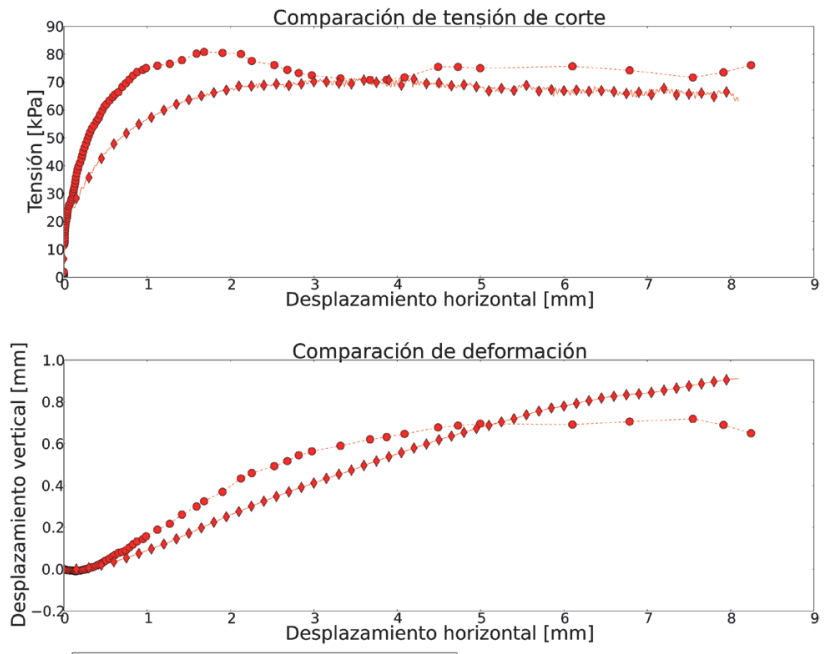

$\cdots$ Lab 80[kPa] Modelo - 80[kPa]

(c)
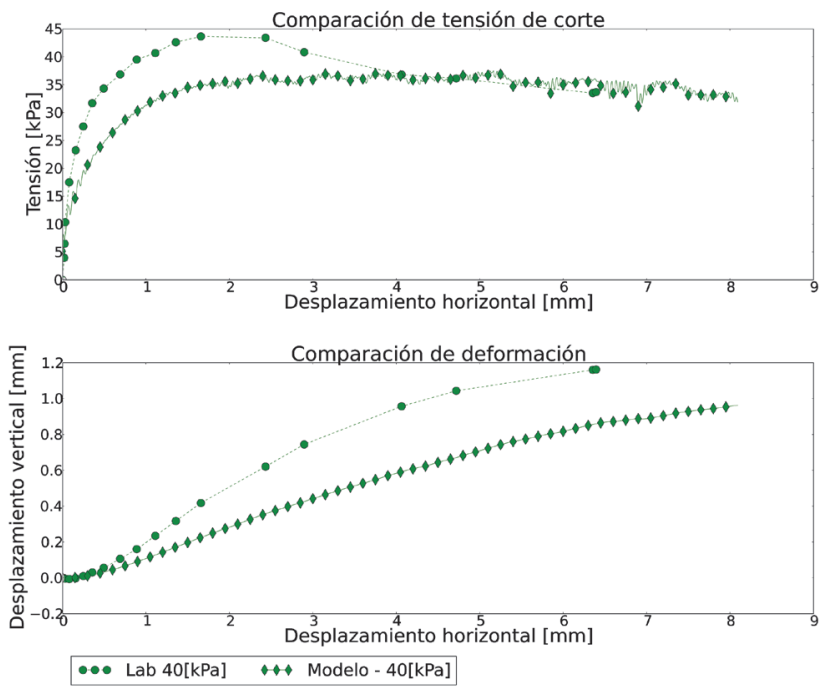

Figura 6: Respuesta del modelo calibrado, a) $160 \mathrm{kPa}$, b) $80 \mathrm{kPa}$ y c) $40 \mathrm{kPa}$ 
laboratorio, pero no se ajusta tan bien como la de tensiones. El aumento volumétrico a grandes deformaciones es altamente influenciado por la forma de las partículas, lo cual es una de las mayores limitantes de este método por el uso de esferas perfectas. En consecuencia, el modelo de fricción a la rotación usado no es capaz de reproducir completamente la transición al estado crítico del material.

La Figura 7 muestra las redes de fuerzas de contactos al final del proceso de asentamiento y al final del corte. Las fuerzas están distribuidas uniformemente al inicio como consecuencia de la presión vertical de confinamiento (Figura 7a). Durante el ensayo, se forma un puntal de suelo diagonal (Figura 7b). Esto se debe a la trabazón entre partículas producto de la geometría y del modelo de fricción a la rotación que dificultan el desplazamiento relativo entre las partículas en la zona central de la probeta.

a)

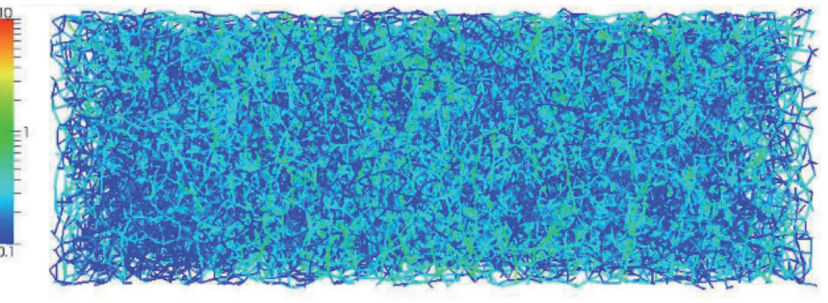

b)

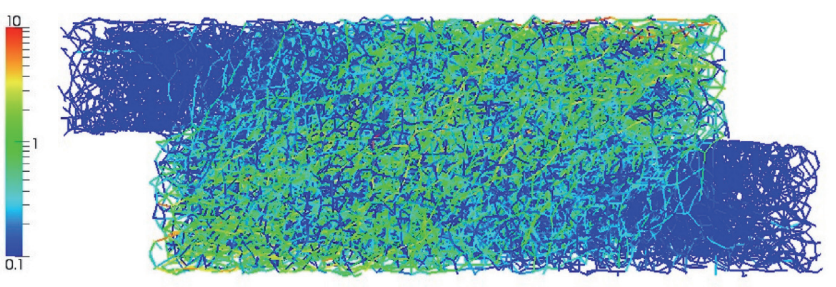

Figura 7: Red de fuerza de contactos a) inicial y b) final

En las Figura 8 y 9, las velocidades de las partículas se promediaron dentro de celdas de $3 \times 1.2 \mathrm{~mm}$ para reducir el número de vectores y representar la velocidad como un campo continuo, conteniendo cada celda la información de alrededor 360 partículas. La Figura 8 muestra una vista lateral (plano que se impone el desplazamiento) del campo de velocidades de las partículas. Al principio del ensayo todas las partículas tienen una velocidad similar, lo que indica que el material se comporta como un continuo (Figura 8a). La gran diferencia de velocidades entre las partículas superiores e inferiores evidencia el desplazamiento relativo entre las partículas en una banda de corte de aproximadamente 2.5 veces el diámetro medio (Figura 8b). (a)

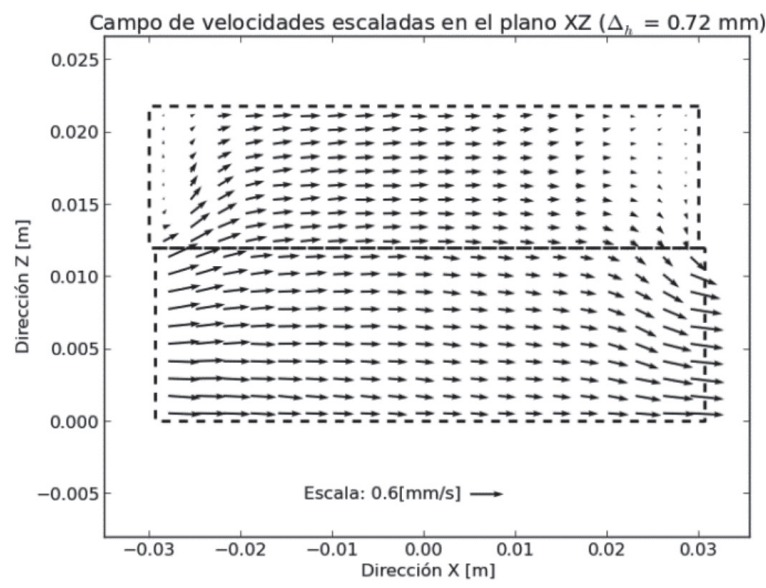

(b)

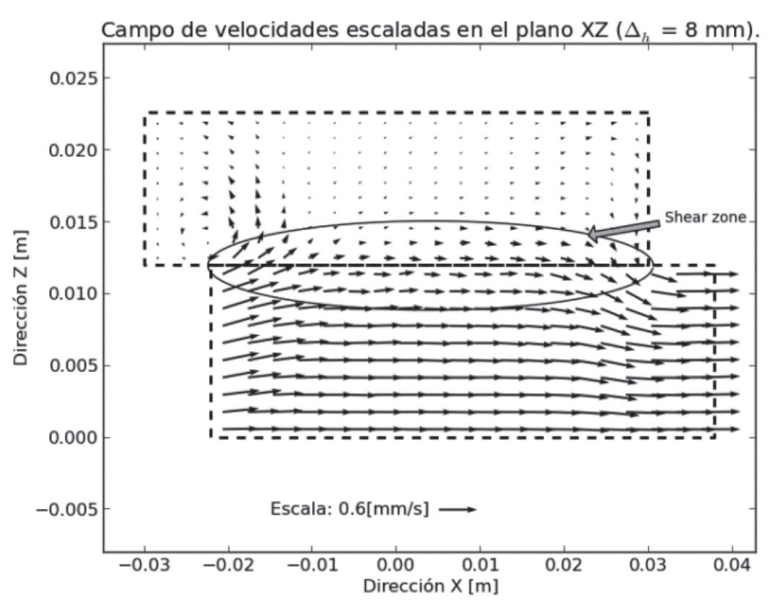

Figura 8: - Campo de velocidades en el plano de corte (plano donde se impone el desplazamiento), a) inicial y b) final

Las partículas no siguen una tendencia clara cuando se ven en el plano normal a la dirección del desplazamiento impuesto (Figura 9), y se mueven con una velocidad 30 veces menor. La existencia de una componente horizontal de velocidad en este plano, indica que las partículas migran de su plano inicial, justificando la implementación de un modelo 3D. Al principio del ensayo las partículas se desplazan verticalmente de forma prácticamente uniforme, con muy poca migración fuera del plano (Figura 9a). Es interesante notar que la migración de partículas no es simétrica, probablemente debido a la generación aleatoria de la posición de las partículas.

\section{Conclusiones}

Cuando se utiliza una metodología discontinua como DEM, la densidad inicial es muy importante y a su vez muy difícil de controlar, comparado con las metodologías continuas. Específicamente, en este trabajo fue muy difícil 
(a)

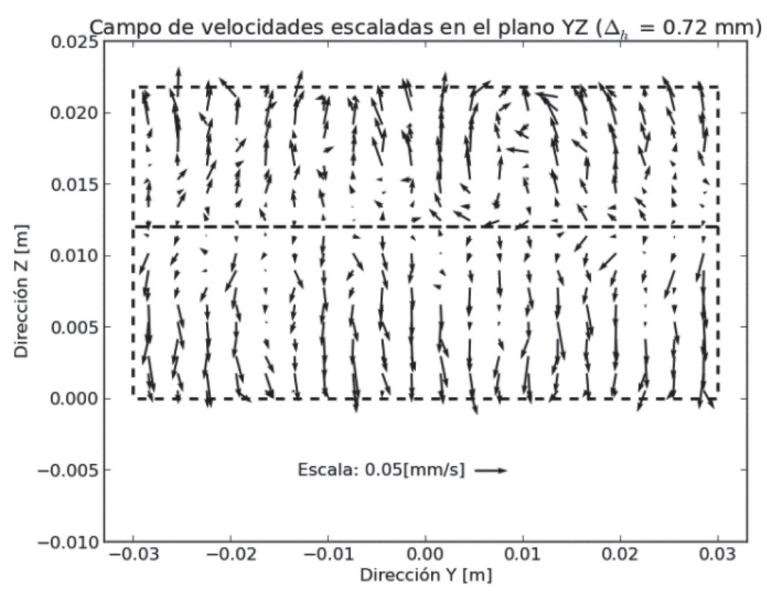

(b)

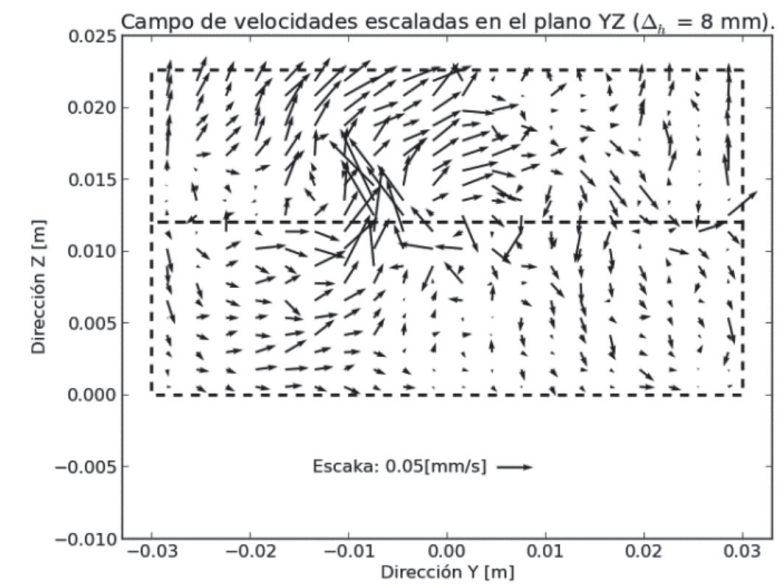

Figura 9: Campo de velocidades fuera del plano de corte, a) inicial y b) final

calibrar los parámetros y la distribución granulométrica adecuada para reproducir conjuntamente el índice de vacíos inicial y la respuesta experimental. Para modelar materiales granulares reales, es imprescindible considerar la forma de la partícula a través de alguna metodología. En este caso, el modelo friccional a la rotación fue adecuado y necesario para simular apropiadamente la respuesta de la arena estudiada. En términos generales, las formas de las curvas obtenidas se acercan bastante a las experimentales. En general, la curva de tensión al corte es cuantitativamente muy similar, mientras que la curva de deformación sólo se asemeja cualitativamente. Con esta metodología de modelación numérica se pueden simular efectos de corte, como se demuestra en la red de contactos. La implementación de un modelo 3D permitió ver migración de partículas fuera del plano del ensayo, por lo que se debe tener mucho cuidado al usar esta y otras metodologías discontinuas en sus respectivas versiones planas.

\section{Agradecimientos}

Este trabajo fue posible gracias al aporte del Fondo Nacional de Desarrollo Científico y Tecnológico FONDECYT 11100157.

\section{Referencias}

Ai, J., Chen, J.-F., Rotter, J.M. and Ooi, J.Y. (2011). Assessment of rolling resistance models in discrete elements simulations. Powder Technology 206(3), 269-282

Fleischamann, J., Plesha, M. and Drugan, W. (2013). Quantitative comparison of two-dimensional and three-dimensional discreteelement simulations of nominally two-dimensional shear flow. International Journal of Geomechanics 13(3), 205-212

Hertz, H. (1882). Über die Berührung fester elastischer Körper. Journal für die reine und angewandte Mathematik 92, 156-171

Kloss, C., Goniva, G., Hager, A., Amberger, S. and Pirker, S. (2012). Models, algorithms and validation for opensource DEM and CFD-DEM. Progress in Computational Fluid Dynamics 12(2/3), 140-152

Li, Y., Xu, Y. and Thornton, C. (2005). A comparison of discrete element simulations and experiments for sandpiles composed of spherical particles. Powder Technology 160(3), 219-228

Mery, D. (2011). BALU: A toolbox Matlab for computer vision, pattern recognition and image processing (http://dmery.ing.puc. cl/index.php/balu)

Mindlin, R.D. (1949). Compliance of elastic bodies in contact. Journal of Applied Mechanics 16, 259-268

Pardo, G. (2013). Estudio experimental y numérico del efecto arco. Tesis de magíster, Pontificia Universidad Católica de Chile

Pardo, G. and Sáez, E. (2014). Experimental and numerical study of the arching effect in coarse sand. Computers and Geotechnics $57,75-84$

Wensrich, C.M. and Katterfeld, A. (2012). Rolling friction as a technique for modelling particle shape in DEM. Powder Technology 217, 409-417 